\title{
Single molecule studies of calix[4]arene-linked perylene bisimide dimers: relationship between blinking, lifetime and/or spectral fluctuations $\dagger$
}

\author{
Abey Issac, ${ }^{a}$ Richard Hildner, ${ }^{a}$ Dominique Ernst, ${ }^{a}$ Catharina Hippius, ${ }^{b}$ \\ Frank Würthner ${ }^{b}$ and Jürgen Köhler ${ }^{*} a$
}

Received 18th April 2012, Accepted 11th June 2012

DOI: $10.1039 / \mathrm{c} 2 \mathrm{cp} 41250 \mathrm{k}$

We recorded fluorescence time traces, and simultaneously either the fluorescence lifetime or the emission spectra from single perylene bisimide (PBI) dimers embedded in a polystyrene matrix. In these traces three distinct intensity levels can be distinguished, which reflect the photo-induced radicalisation of one of the perylene subunits. Differences in the energy transfer rate between the neutral PBI and the reversibly formed radical anion give rise to variations in the chronological order of the appearance of the intensity levels, which allowed us to categorise the time traces into three distinct groups: Type 1 blinking corresponds to a high energy transfer rate, type 2 blinking to fluctuations between large and small transfer rates (dynamic quenching), and type 3 blinking results from small energy transfer rates together with Coulomb blockade. The information that we obtain from the distributions of the fluorescence lifetimes at the various signal levels allows us to relate these differences to properties of the local polymer environment of the dimers.

\section{Introduction}

The development of next-generation organic devices with novel properties is driven by the ability to assemble organic molecular building blocks into supramolecular architectures. ${ }^{1-10}$ Particularly perylene bisimides (PBIs) have attracted considerable attention over the past few years because of their favourable photophysical properties: they possess a large absorption cross section in the visible spectral range, a high emission quantum yield close to unity, and an outstanding photochemical stability. ${ }^{11-18}$ Moreover, their optical and electronic properties can be easily tuned by appropriate substitution at the bay positions. ${ }^{19,20}$ Novel assemblies based on PBIs have a great potential for a wide range of applications such as in artificial light harvesting, ${ }^{1,4,13}$ organic thin-film transistors ${ }^{9}$ and organic light emitting diodes. ${ }^{6}$

For a variety of applications, the molecular building blocks have to be embedded in flexible matrices, for example in polymer films, and the devices operate under ambient conditions. However, the characterization of their optical and electronic properties under these conditions is hampered by ensemble techniques, because each chromophore experiences a slightly different local environment giving rise to variations in the interaction strength with the surrounding and in the

\footnotetext{
${ }^{a}$ Experimental Physics IV and Bayreuth Institute for Macromolecular Research (BIMF), University of Bayreuth, 95440 Bayreuth,

Germany.E-mail: juergen.koehler@uni-bayreuth.de;

Fax: + 4992155 4002; Tel: + 49921554001

${ }^{b}$ Universität Würzburg, Institut für Organische Chemie and Center for Nanosystems Chemistry, Am Hubland, 97074 Würzburg, Germany

$\dagger$ Electronic supplementary information (ESI) available: See DOI: $10.1039 / \mathrm{c} 2 \mathrm{cp} 41250 \mathrm{k}$
}

energies of the optical transitions. Single molecule fluorescence spectroscopy, which is free from ensemble averaging, has been demonstrated to be a unique method for investigating the excited state dynamics in various heterogeneous multichromophoric structures. ${ }^{1,7,8,14-16,21-26}$ Recent single molecule studies of $\pi$-stacked PBI dimers and trimers embedded in polymer matrices revealed conformational changes due to the interactions with the polymer matrix by recording variations of fluorescence intensities and excited state lifetimes. ${ }^{16,27-29}$

Generally, the fluorescence signal of single quantum emitters, such as semiconductor quantum dots or organic molecules, is characterized by a random succession of bright (emitting) and dark (non-emitting) periods, which is called intermittency or blinking. Today, it is generally accepted that the underlying mechanism is charging or charge separation occurring in the single emitter. ${ }^{30-37}$ For organic molecules, the reversible formation of a non-fluorescent radical species upon photoexcitation is considered to be the origin of long-lived dark states ( $\mu$ s to $s$ timescale). In a disordered medium with a nearly continuous distribution of trapping sites for the charges the distribution of the duration of such long-lived dark states (usually referred to as off-time distribution) typically follows an inverse power-law behaviour over several decades in probability and time. ${ }^{33-35,38,39}$ We note, however, that a reduction of the heterogeneity of the local environment of the emitter can give rise to a stretched or even a single exponential off-time distribution. ${ }^{40}$ Despite many studies there is still little knowledge about, for example, the electrostatic interaction of the radical ion with the local environment, spatial redistribution of the charge density in the radical ion and the nature of the irreversible photoproduct, 
because such dark states cannot be observed directly by optical spectroscopy. However, having a second chromophore as a reporter in close vicinity of the dark species may provide insights into the nature of these states. Therefore, blinking studies of multichromophoric systems as such is interesting because the neutral bright (emitting) unit(s) carry information about the nearby charged dark (non-emitting) radical unit(s) that can be read out by recording the fluorescence intensity, excited state lifetime and emission spectra.

In a previous paper we have studied the fluorescence intermittency of single dimers consisting of two identical, covalently linked PBI derivatives that were immobilized in a polystyrene (PS) film. ${ }^{41}$ These weakly coupled dimers featured fluorescence blinking between three distinct intensity levels called dimer, monomer and background level. The dimer level was attributed to the emission from both neutral PBI units, which can be described as two independent emitters due to the weak electronic coupling; the monomer level was ascribed to the emission from one of the PBI units, while the other monomer is reversibly transformed to the radical anion, $\mathrm{PBI}^{-}$due to the electron affinity of the $\mathrm{PBI}^{42}$ or irreversibly photobleached. The signal level drops permanently to the background level when both PBI monomers are photobleached. According to their chronological order the intensity traces were categorized into three distinct types prior to the irreversible reduction of the signal to the monomer level: Type 1 traces featured blinking between the dimer and the background level. For type 2 blinking the signal fluctuated randomly from the dimer level to both the monomer and the background level. Finally, type 3 traces showed blinking only between the dimer and the monomer level. These findings were explained by a model that related the variations in blinking behaviour to different regimes of electronic excitation energy transfer from the neutral $\mathrm{PBI}$ to the $\mathrm{PBI}^{-}$. The idea was that the energy transfer rate is the highest for type 1 blinking. Fluctuations between large and small transfer rates (dynamic quenching) or alternatively a small transfer rate without Coulomb blockade were suggested to cause type 2 blinking, whereas type 3 blinking was attributed to dimers that exhibited a small energy transfer rate together with Coulomb blockade.

In this paper, we substantiate this blinking model by recording simultaneously with the blinking trajectories either the fluorescence lifetime or the emission spectra of single dimers. The lifetime data from different signal levels for type 2 dimers suggest that dynamic quenching is the most likely process. For type 3 dimers the comparison of spectral information in the different signal levels provides evidence for the presence of a Coulomb blockade. The observation of charge-induced enhanced spectral diffusion also gives information about the localisation of the charge on the dark $\mathrm{PBI}^{-}$and the irreversible photoproduct, which is usually impossible to detect optically. Finally, we provide a picture of how the various regimes of energy transfer rates can be related to differences of the polystyrene free volume around the dimers.

\section{Experimental section}

Perylene bisimide (PBI) dimers were synthesised and characterized as detailed previously. ${ }^{43}$ The dimer consists of a tetrakis(propyloxy)-calix[4]arene scaffold and two PBI chromophores that are functionalised with four tert-butylphenoxy subunits in the bay area. The molecular structure based on X-ray crystallography and molecular modelling is shown in Fig. $1{ }^{43}$ For the single-molecule experiments, the PBI-dimers were dissolved at a concentration of $4 \mu \mathrm{M}$ in toluene, which served as a stock solution. Small aliquots of this solution were further diluted in a polystyrene-toluene mixture $\left(10 \mathrm{mg} \mathrm{ml}^{-1}\right)$ to achieve a PBI concentration of $10^{-10} \mathrm{M}$. Subsequently, $30 \mu 1$ of this mixture was spin coated on a freshly cleaned glass cover slip at $2500 \mathrm{rpm}$ for $60 \mathrm{~s}$.

The samples were excited either with a continuous-wave laser (Monopower 532-100-SM, Alphalas GmbH, Germany) at $532 \mathrm{~nm}$ with an excitation intensity of $6 \mathrm{~kW} \mathrm{~cm}^{-2}$ in the excitation focus, or with a diode laser (LDH-P-C-450B, PicoQuant) at $450 \mathrm{~nm}$ providing pulses with a width of $70 \mathrm{ps}$ (FWHM) at a repetition rate of $20 \mathrm{MHz}$. The power of this laser was adjusted to a value that corresponded to the same timeaveraged intensity in the focus as for the continuous excitation. The excitation light was directed into a home-built confocal microscope and reflected by a dichroic beam splitter (z532RDC, AHF for the spectrally resolved experiments and 500DCXR,

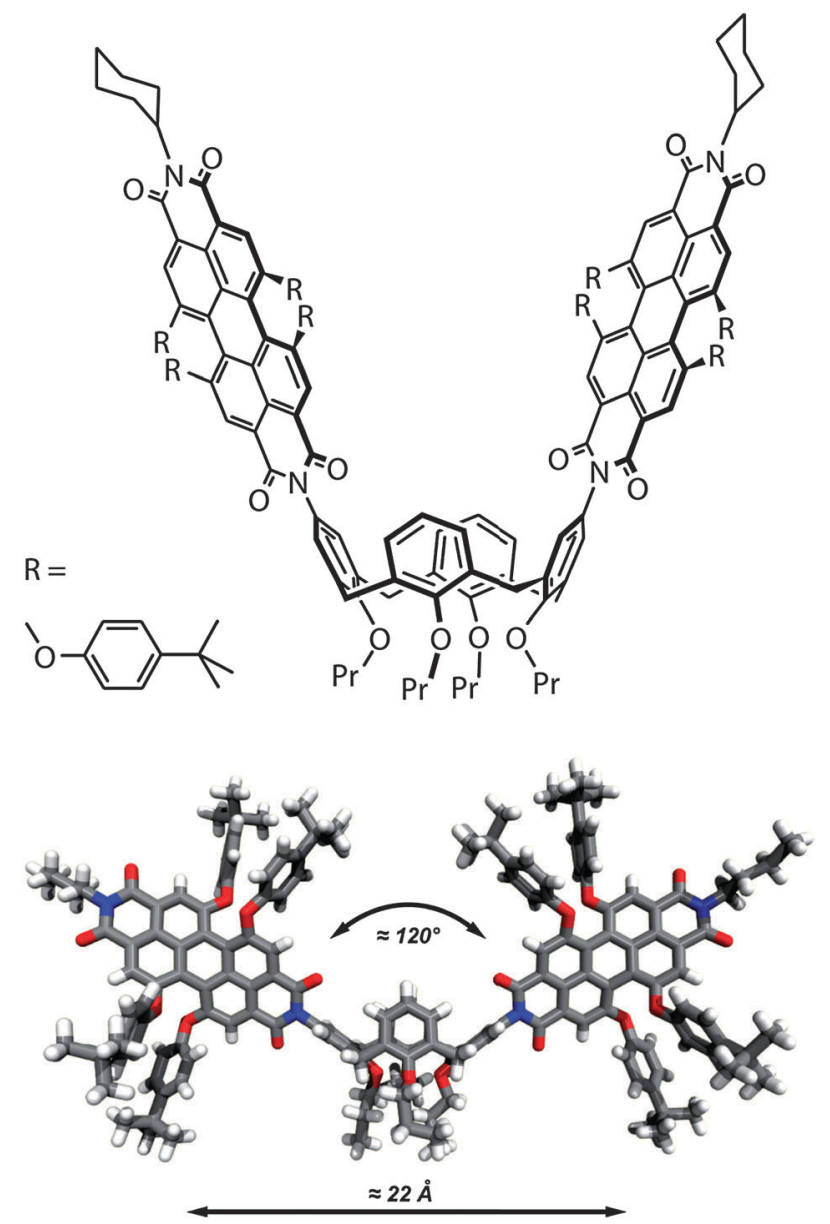

Fig. 1 Molecular structure of the calix[4]arene tethered perylene bisimide dimer (top) and its conformation found in a single crystal (bottom). ${ }^{43}$ The two PBI chromophores are covalently linked through a tetrakis(propyloxy)-calix[4]arene. The angle between the long axis transition-dipole moments of the two perylene bisimide chromophores is about $120^{\circ}$ and the distance between the centres of the chromophores is about $22 \AA$. 
AHF for the time-resolved experiments) towards an infinitycorrected oil-immersion objective (PlanApo, 60x, NA $=1.45$, Olympus). The emission from a single dimer was collected by the same objective and passed through the dichroic beam splitter and a dielectric filter (545 LP, AHF).

For the spectrally resolved experiment, the signal was focused on the entrance slit of a spectrometer (SPEC 250IS, Bruker Optics Inc.) that is equipped with a grating (150 grooves per $\mathrm{mm}$ ) blazed at $500 \mathrm{~nm}$, providing a spectral resolution of $1 \mathrm{~nm}$ at $600 \mathrm{~nm}$ (corresponding to $30 \mathrm{~cm}^{-1}$ ). The spectrally dispersed signal was detected with a back-illuminated electron-multiplying charge-coupled device (emCCD, iXon DV887-BI, Andor Technology). Typically, 600 fluorescence spectra were registered successively from each dimer with an exposure time of $100 \mathrm{~ms}$. The fluctuations of the fluorescence intensity as a function of time are obtained by integrating the intensity of the individual spectra. This approach yields simultaneously the fluorescence intensity and the peak emission wavelength as a function of time.

Alternatively, the emission signal is focused on a singlephoton avalanche photodiode (Micro Photon Devices) and the electrical signal from the detector is split into two components. One component is fed into a counting card (MCA-3 Series/P7882, FASTComTec GmbH) to record the fluorescence intensity as a function of time. The other component is used to start a time-correlated single-photon counting (TCSPC) module (TimeHarp 200, PicoQuant). Each fluorescence decay curve is integrated for $100 \mathrm{~ms}$ with a temporal resolution of $300 \mathrm{ps}$. The total duration of these experiments was $64 \mathrm{~s}$. This scheme allows for the simultaneous registration of the fluorescence intensity and the fluorescence lifetime as a function of time. All experiments were carried out at room temperature.

\section{Results}

The fluorescence intensity from individual PBI dimers shows on/off blinking as a function of time which is a well-known fingerprint of single-molecule detection. The recorded time traces have in common that they feature three distinct intensity levels: a high level, a low level at about half of the high-level intensity, and the background level. The signal levels are discriminated by setting two thresholds for the recorded intensities (Fig. 2, dashed lines). The lower threshold discriminates between the background and the low-signal level, and the upper threshold discriminates between the low- and the high-signal level. Based on the chronological order of the fluctuations between these three intensity levels, the time traces can be grouped into three different types of blinking behaviour.

Examples of type 1 blinking are presented in Fig. 3, where the traces initially show blinking between the high signal level and the background level. At later times, the signal is reduced to the low level during the on-times. Fig. 3a-d show an example where we recorded the blinking as well as the emission spectra of the PBI dimers. Each emission spectrum (Fig. 3b and c) is fitted with the sum of two Gaussian functions, one for the purely electronic transition $(0-0)$ and one for the vibrational side band; the peak emission wavelength is extracted from the maximum of the fitted $0-0$ band, Fig. 3d (see Fig. S1 (ESI $\dagger$ ) for details). As long as the fluorescence intensity corresponds to the high intensity level (red), the peak emission

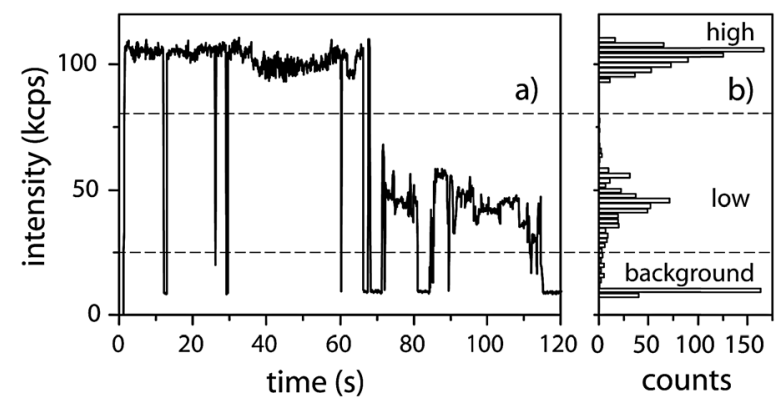

Fig. 2 (a) Typical intensity trajectory of a single PBI dimer molecule in a polystyrene matrix recorded with a bin time of $100 \mathrm{~ms}$ and (b) a histogram of the observed emission intensities. The dotted lines represent the thresholds used to discriminate between high/low and low/background signal levels, respectively.
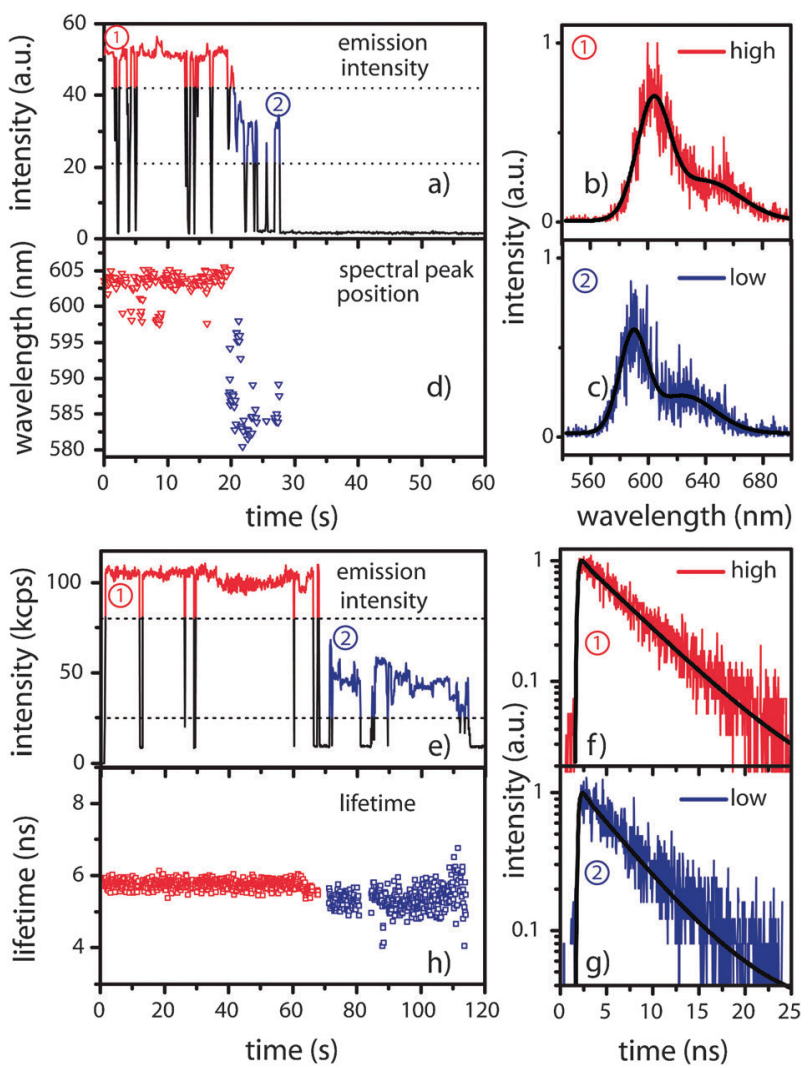

Fig. 3 (a) Type 1 intensity trajectory of a single dimer recorded together with the emission spectrum. The dotted lines represent the intensity threshold levels. The colours encode the high-signal (red), the low signal (blue), and the background level as well as blinking events faster than the bin time (black). (b), (c) Examples of fluorescence spectra recorded during the high (b) and low (c) signal levels. The corresponding intervals are indicated as 1 and 2 in (a), respectively. Each spectrum can be fitted with the sum of two Gaussians (black solid line). (d) Variation of the spectral peak position of the emission spectrum for the trajectory shown in (a). (e) Type 1 intensity trajectory of a single dimer recorded together with the fluorescence lifetime. (f), (g) Semilogarithmic representations of representative fluorescence transients recorded during the high (f) and the low (g) signal levels indicated as 1 and 2 in (e), respectively. The decays can be fitted by a monoexponential function (black solid line). (h) Variation of the fluorescence lifetime for the trajectory shown in (e). 
wavelength is centred at about $603 \mathrm{~nm}$ and shows only small variations between $597 \mathrm{~nm}$ and $605 \mathrm{~nm}$. Once the signal decreased to the low signal level (blue), the peak position of the emission is centred at about $586 \mathrm{~nm}$ and features strong fluctuations covering the range from 580 to $598 \mathrm{~nm}$. Interestingly, the shape of the emission spectrum is not affected by these fluctuations. Fig. 3e-h shows a dimer molecule where we recorded simultaneously the blinking and the fluorescence lifetime. All transients (Fig. $3 \mathrm{f}$ and g) are compatible with a monoexponential decay. For this example, we find a mean fluorescence lifetime of $5.8 \mathrm{~ns}$ which varies between $5.4 \mathrm{~ns}$ and $6.1 \mathrm{~ns}$ during the high-intensity periods (red). During the low intensity periods (blue) the mean fluorescence lifetime is $5.3 \mathrm{~ns}$ and varies between 4 ns and 6.8 ns, Fig. $3 \mathrm{~h}$.

The blinking trajectories of type 2 are characterized by fluctuations from the high signal level to both the low and the background level, as well as recoveries from the low and the background level to the high signal level. Examples are displayed in Fig. 4a and c together with the variations in either the spectral peak position (b) or the fluorescence lifetime (d). For the low-intensity periods we distinguish those in-between high-intensity periods (green) and those that are not terminated by a high-intensity period at the end of the trajectory (blue). For the dimer molecule shown in Fig. $4 \mathrm{a}$ and $\mathrm{b}$ the peak emission wavelength is centred around $595 \mathrm{~nm}$ and shows only small variations during the intensity fluctuations. Only at the end of the experiment strong fluctuations between $592 \mathrm{~nm}$ and $607 \mathrm{~nm}$ are observed. For the other example, Fig. 4c and d, we find during the high-intensity periods (red) a mean fluorescence lifetime of $6.3 \mathrm{~ns}$ which varies between $5.3 \mathrm{~ns}$ and $7.8 \mathrm{~ns}$. For the low-intensity periods in-between the high-intensity intervals we find a mean of 6.6 ns which varies strongly between $4.3 \mathrm{~ns}$ and $8.0 \mathrm{~ns}$ (green), and for the low-intensity periods at the end of the trajectory the mean fluorescence lifetime is $6.1 \mathrm{~ns}$ which ranges from $4.3 \mathrm{~ns}$ to $8.3 \mathrm{~ns}$ (blue). In our previous work ${ }^{41}$ we have proposed a "dynamic quenching model", i.e. fluctuations between large and small $\mathrm{PBI} \rightarrow \mathrm{PBI}^{-}$ transfer rates, for type 2 dimers in the low-intensity periods in-between the high-intensity intervals (green in Fig. 4c and d). This dynamic quenching would be reflected in a clear correlation between the fluorescence intensity and the lifetime, which we indeed observe (see Fig. S2a and S2b, ESI $\dagger$ ).

Finally, type 3 blinking features time traces where the blinking occurs only between the high and the low signal levels prior to a reduction of the signal to the low signal level during the on times. Typical examples are shown in Fig. 4e and g. As long as the high signal level is registered, the peak emission wavelength is centred at $601 \mathrm{~nm}$ and varies between $599 \mathrm{~nm}$ and $603 \mathrm{~nm}$ (Fig. 4e and f; red). Once the signal drops to the low signal level in-between the high signal levels the peak emission wavelength is shifted to $605 \mathrm{~nm}$ and fluctuates between $603 \mathrm{~nm}$ and $606 \mathrm{~nm}$ (green). Later the molecule shows blinking only between the low level and the background level. During this period the peak emission wavelength is centred at $603 \mathrm{~nm}$ which varies between $596 \mathrm{~nm}$ and $606 \mathrm{~nm}$ (blue), Fig. $4 \mathrm{e}$ and $\mathrm{f}$. The fluorescence lifetime during the high signal levels features a mean value of $5.6 \mathrm{~ns}$ which varies between $5 \mathrm{~ns}$ and $5.9 \mathrm{~ns}$ (Fig. $4 \mathrm{~g}$ and h; red). Similar values are found during the long low-signal level towards the end of the observation time, i.e. a mean of about $5.1 \mathrm{~ns}$ that changes between $4.5 \mathrm{~ns}$ and 5.9 ns (blue). Interestingly, these numbers are distinctly different from those observed for the low signal levels that
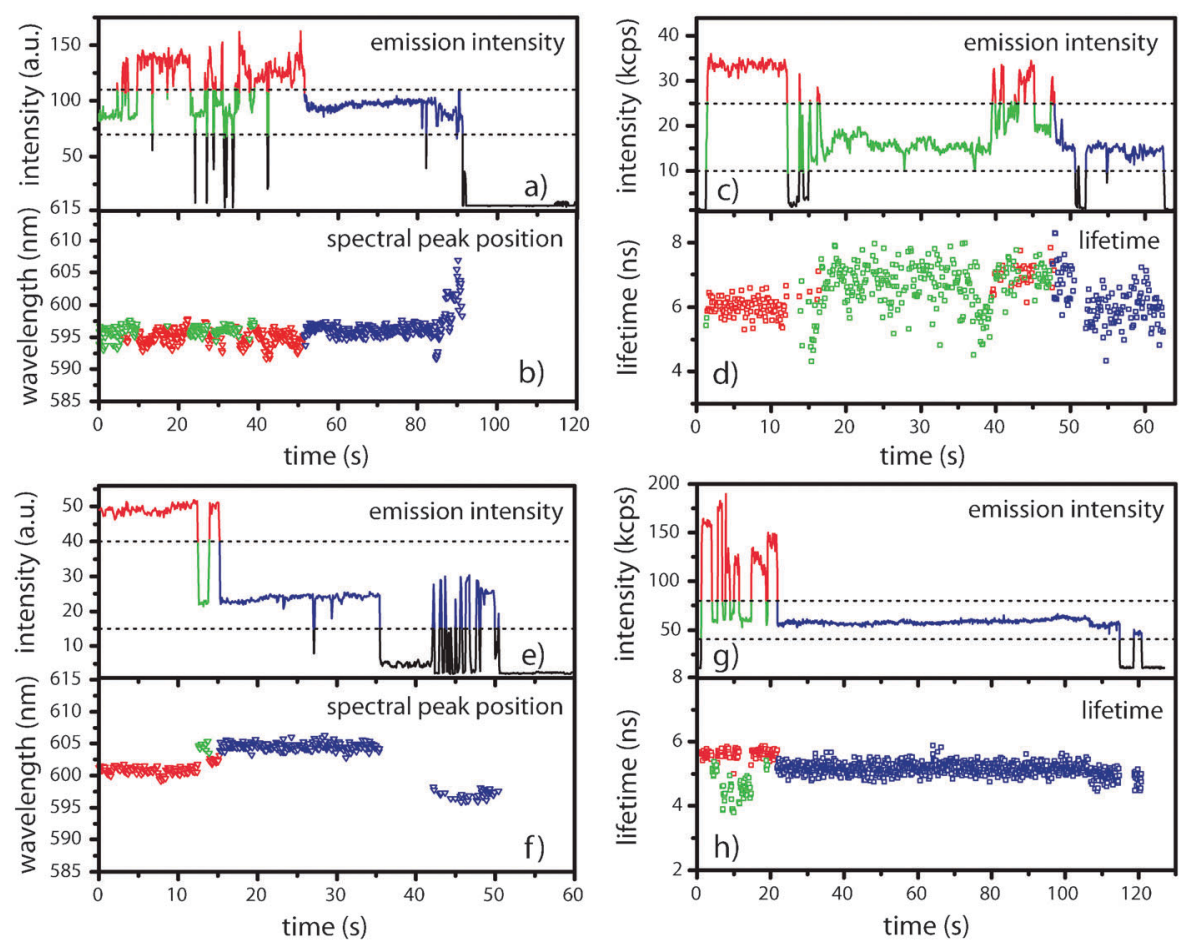

Fig. 4 (a)-(d) Type 2 intensity trajectories (a), (c), spectral peak position (b) and lifetime (d). (e)-(h) Type 3 intensity trajectories (e), (g), spectral peak position (f) and lifetime (h). The colours encode the high-signal level (red), the low-signal level in-between two high-signal levels (green), the low-signal level not terminated by a high-signal level (blue), and the background level as well as blinking events faster than the bin time (black). 
occur in-between two high signal periods (green). There we found a mean value of $4.6 \mathrm{~ns}$ and variations between $3.8 \mathrm{~ns}$ and 5.4 ns, Fig. $4 \mathrm{~g}$ and $\mathrm{h}$.

In total, we studied 44 dimers and the appearance of type 1 : type 2 : type 3 blinking behaviour was about equally distributed $(36 \%: 32 \%: 32 \%)$. This proportion is different from our earlier work $(62 \%: 19 \%: 16 \%)^{41}$ which might be due to two reasons: First, here we use a higher excitation wavelength as compared to our previous study $(532 \mathrm{~nm}$ here vs. $514 \mathrm{~nm}$ in ref. 41), which reduces the excess energy available to a second electron to overcome the electrostatic energy barrier in the $\mathrm{PBI}-\mathrm{PBI}^{-}$system. Hence, the probability of Coulomb blockade for type 3 dimers (refer Section 4.2) is increased in our present study. Second, there may be statistical limitations due to the smaller number of single molecules investigated here (44 vs. 193 in ref. 41).

Inspired by the finding that the fluorescence lifetimes during the low signal periods in-between two high-signal periods (those prior to irreversible photobleaching of one of the PBI units) are substantially different from those found after photobleaching of one of the monomers, we analysed the data sets separately for the following signal levels: The high-signal level, which will be referred to as the dimer level D (red), the low signal level that occurs in-between high-signal periods, which will be termed intermediate level IM (green), and the lowsignal level after irreversible photobleaching of one of the monomers, which will be named monomer level M (blue).

The distributions of the fluorescence lifetimes for the various blinking categories and signal levels are shown in Fig. 5a-d. For emission from the D level (red bars) the statistical parameters [mean, standard deviation (SD)] of the fluorescence lifetime distributions are $5.8 \mathrm{~ns}(0.67 \mathrm{~ns})$ for type $1,5.4 \mathrm{~ns}$ $(0.85 \mathrm{~ns})$ for type 2 , and $6.0 \mathrm{~ns}(0.6 \mathrm{~ns})$ for type 3 , respectively. The type 2 dimer histogram (Fig. 5b) appears broader with respect to those of the other two types (Fig. 5a and c). For emission from the IM level the lifetime distributions differ significantly between type 2 and type 3 (Fig. 5b and c green open bars). For type 2 IM emission the histogram features many entries between $1.5 \mathrm{~ns}$ and $4 \mathrm{~ns}$ resulting in a mean (SD) of $4.9 \mathrm{~ns}$ (1.42 ns), whereas the lifetime distribution found for the type $3 \mathrm{IM}$ emission features a mean (SD) of $5.9 \mathrm{~ns}(0.97 \mathrm{~ns})$. Fig. $5 \mathrm{~d}$ presents the lifetime distributions for emission from the $\mathrm{M}$ levels for the three different types of blinking categories. The distribution for type 1 and type 3 are statistically identical whereas that for type 2 is shifted to longer lifetimes. The statistical parameters (mean, SD) are $5.2 \mathrm{~ns}, 0.62 \mathrm{~ns}$ (type 1); $6.1 \mathrm{~ns}, 0.71 \mathrm{~ns}$ (type 2); and $5.5 \mathrm{~ns}, 0.61 \mathrm{~ns}$ (type 3), respectively. In summary, the most striking finding from Fig. 5a-d is the clear deviation of the IM level histograms from all other distributions.

We further compared the distributions of the linewidths of the emission spectra, which we obtain from the full width at half maximum (FWHM) of the fitted $0-0$ transitions, Fig. 5e-h. The setup of the figure is similar to that of Fig. 5a-d. For emission from the D level, the distributions of the bandwidths are not significantly different among the three categories (Fig. 5e-g, red bars). We find a mean (SD) of $621 \mathrm{~cm}^{-1}\left(47 \mathrm{~cm}^{-1}\right)$ for type $1,626 \mathrm{~cm}^{-1}\left(51 \mathrm{~cm}^{-1}\right)$ for type 2 , and $608 \mathrm{~cm}^{-1}\left(60 \mathrm{~cm}^{-1}\right)$ for type 3 , respectively. For types 2 and 3, we obtain additional bandwidth histograms for the emission from the IM levels (Fig. 5f and g green open bars). Strikingly, these distributions are shifted with respect to each other, which is reflected by the mean (SD) of $609 \mathrm{~cm}^{-1}$ $\left(52 \mathrm{~cm}^{-1}\right)$ for type 2 and $645 \mathrm{~cm}^{-1}\left(49 \mathrm{~cm}^{-1}\right)$ for type 3 . For comparison Fig. $5 \mathrm{~h}$ shows the respective distributions for emission from the $\mathrm{M}$ level for all blinking types. While the distributions are not significantly different with respect to their mean (SD) [type 1: $600 \mathrm{~cm}^{-1}\left(52 \mathrm{~cm}^{-1}\right)$; type 2: $580 \mathrm{~cm}^{-1}$ $\left(50 \mathrm{~cm}^{-1}\right)$; type $\left.3: 615 \mathrm{~cm}^{-1}\left(51 \mathrm{~cm}^{-1}\right)\right]$, the histogram for type 3 shows a clearly shifted peak value of $645 \mathrm{~cm}^{-1}$ with respect to the peak value of $585 \mathrm{~cm}^{-1}$ found for the other two histograms.

To probe a possible correlation between the spectral peak positions of the $(0-0)$ emission band and the blinking behaviour, we compared the distributions of the $(0-0)$ peak emission energies for all three types (see Fig. S3, ESI $\dagger$ ). The histogram covers a range of about $1200 \mathrm{~cm}^{-1}$ which provides a measure for the static heterogeneity of the sample.
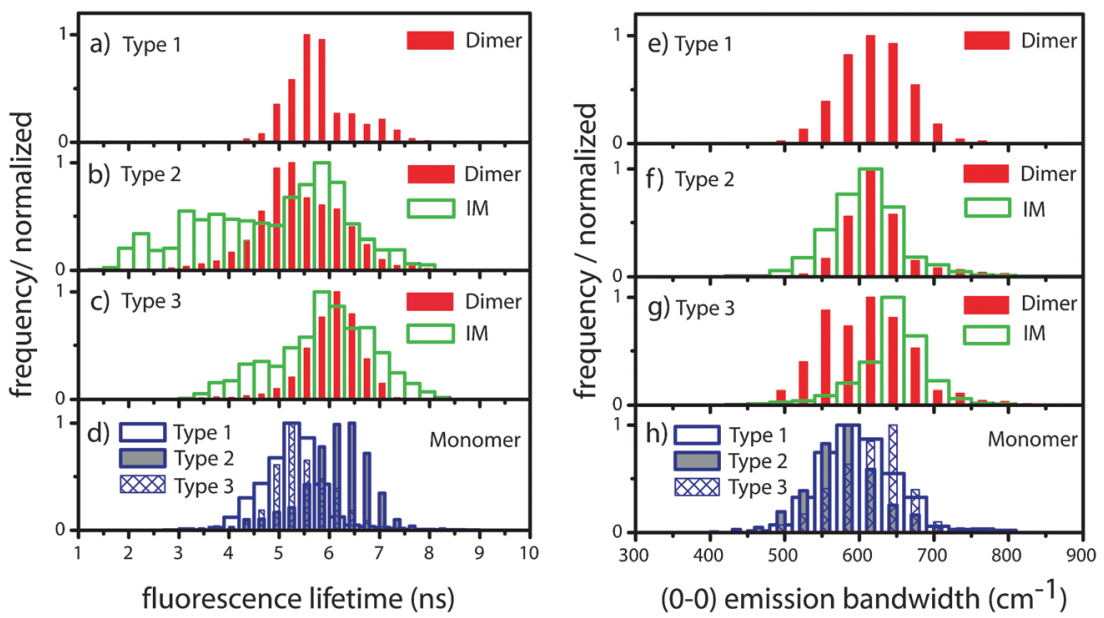

Fig. 5 Distributions of the fluorescence lifetimes (a)-(c) and the linewidths (FWHM) (e)-(g) of the (0-0) emission band for type 1, type 2, and type 3 blinking for the dimer level (D, red) and the intermediate level (IM, green) respectively. Distributions of the lifetimes (d) and the linewidths (h) of the (0-0) emission band for the monomer level (M) for the three types of blinking. 
Table 1 Photophysical parameters of PBI dimers embedded in PS matrix. The mean (standard deviation, SD) is calculated from the data shown in Fig. 5. $\delta E$ is calculated as described in the text

\begin{tabular}{|c|c|c|c|c|c|c|c|c|c|}
\hline & \multicolumn{3}{|c|}{$\delta E^{(\text {level })}\left(\mathrm{cm}^{-1}\right)$} & \multicolumn{3}{|c|}{ Mean (SD) of $(0-0)$ emission bandwidth $\left(\mathrm{cm}^{-1}\right)$} & \multicolumn{3}{|c|}{ Mean (SD) of fluorescence lifetime (ns) } \\
\hline & Type 1 & Type 2 & Type 3 & Type 1 & Type 2 & Type 3 & Type 1 & Type 2 & Type 3 \\
\hline Dimer (D) & 46 & 42 & 49 & $621(47)$ & $626(51)$ & $608(60)$ & $5.8(0.67)$ & $5.4(0.85)$ & $6.0(0.60)$ \\
\hline Intermediate (IM) & - & 49 & 81 & - & $609(52)$ & 645 (49) & - & $4.9(1.42)$ & $5.9(0.97)$ \\
\hline Monomer (M) & 84 & 85 & 87 & $600(52)$ & $580(50)$ & $615(51)$ & $5.2(0.62)$ & $6.1(0.71)$ & $5.5(0.61)$ \\
\hline
\end{tabular}

However, the entries for the different types of blinking strongly overlap and it is clear that the peak position of the emission do not correlate with the blinking behaviour.

The data presented in Fig. 3 and 4 show that the peak emission energy of the recorded spectra fluctuates as a function of time (spectral diffusion slower than the bin time of $100 \mathrm{~ms}$ ). This is related to the dynamic heterogeneity in the sample. In order to quantify the spectral diffusion at the different intensity levels, a parameter $\delta E^{(\text {level })}$ is defined:

$$
\delta E^{(\text {level })}=\frac{\sum_{i} \mathrm{SD}_{i}^{(\text {level })} \cdot N_{i}^{(\text {level })}}{\sum_{i} N_{i}^{(\text {level })}}
$$

where $\mathrm{SD}_{i}^{(\text {level) }}$ is the standard deviation of the distribution of the $(0-0)$ peak emission energies at a certain signal level (D, IM or $\mathrm{M}$ ) for the $i$ th molecule (see Fig. S1b, ESI $\dagger$ ). $N_{i}^{(\text {level) }}$ represents the number of spectra at that particular signal level. Accordingly, $\delta E^{(\text {level })}$ is a weighted mean of the standard deviation of the distribution of the peak emission energies and gives a measure for the spectral diffusion in the various signal levels of the three blinking types, as summarized in Table 1. No significant differences between the three types of blinking are found for $\delta E^{(\mathrm{D})}\left(42 \mathrm{~cm}^{-1}\right.$ to $\left.49 \mathrm{~cm}^{-1}\right)$ and $\delta E^{(\mathrm{M})}$ $\left(84 \mathrm{~cm}^{-1}\right.$ to $\left.87 \mathrm{~cm}^{-1}\right)$. Yet, a clear difference for this parameter is found between the IM levels of type $2\left(\delta E^{(\mathrm{IM})}=49 \mathrm{~cm}^{-1}\right)$ and type $3\left(\delta E^{(\mathrm{IM})}=81 \mathrm{~cm}^{-1}\right)$ blinking.

\section{Discussion}

It has been shown previously that the electronically excited states of the PBI dimers can be treated in the weak coupling limit. ${ }^{14,15,24,25,41}$ In short, the distribution of the excitation energy over the dimer molecule is determined by the ratio of the transition dipole-dipole interaction $(V)$ and the energetic mismatch of the electronic excitation energies of the two monomer units (4). Assuming a transition-dipole moment of the PBI-monomer in the order of 10 Debye and taking the geometry of the dimer as shown in Fig. 1 into account, we estimate a value of $40 \mathrm{~cm}^{-1}$ for V. ${ }^{17,41,44}$ For organic molecules embedded in a polymer matrix, a modulation of the electrostatic interaction between the molecule and the local surrounding may cause $\Delta$ to be as high as several hundred wavenumbers. ${ }^{14,15,45,46}$ Accordingly, $V / \Delta \ll 1$ and it is therefore justified to assume that the excitations are localised on the subunits of the PBI-dimers. As a consequence of this, the dimer level (D) is assigned to the emission from a single dimer that can be described as two independent emitters, which is reflected in our data in the slightly larger $(0-0)$ emission linewidths for the spectra recorded in the $\mathrm{D}$ level with respect to the $\mathrm{M}$ level (Fig. $5 \mathrm{e}-\mathrm{h}$ ). The intermediate level (IM) is attributed to the emission from one of the monomer units while the other one turned reversibly to a non-fluorescent state. After irreversible photobleaching of one of the PBI units the monomer level (M) is observed, which is associated with the emission from the remaining monomer unit. Finally, upon irreversible photobleaching of the second PBI unit of the dimer the signal drops permanently to the background level (B).

A generally accepted mechanism for the fluorescence blinking of single quantum emitters (e.g. quantum dots, organic molecules) is the transition between a neutral bright state and a charged dark state. ${ }^{30,31,33,34}$ For the electron-poor PBI chromophores the charged state is presumably a radical anion, $\mathrm{PBI}^{-42}$ that can be formed in two ways: First, there can be a charge tunnelling process between the PBI and the PS matrix. Second, upon photoexcitation a charge transfer state $\left(\mathrm{PBI}-\right.$ calixarene $\left.{ }^{+}-\mathrm{PBI}^{-}\right)$ can be created ${ }^{43}$ followed by a reduction of calixarene ${ }^{+}$to its neutral state by charge transfer from the matrix. Although from our data we cannot distinguish these two options for the initial formation of $\mathrm{PBI}^{-}$, the subsequent neutralization of the $\mathrm{PBI}^{-}$ unit has to be caused by charge tunnelling between PBI and the heterogeneous surrounding matrix, because we have observed a power-law blinking statistics on these dimer molecules, ${ }^{41}$ indicating a broad spatial distribution of trapping sites. In contrast, a neutralisation of $\mathrm{PBI}^{-}$via charge transfer with calixarene $^{+}$would lead to an exponential 'off'-time distribution, which we did not detect. Our interpretation of the three different types of blinking is summarised in Fig. 6 (see also ref. 41), and the following scenarios can be distinguished prior to irreversible photobleaching of one of the monomers:

During type 1 blinking, the intensity fluctuates between the dimer level (D) and the background level (B), see Fig. 6a. The reversible photoreduction of one of the PBIs completely quenches the emission from the remaining neutral monomer by excitation energy transfer to the radical that is fast with respect to the fluorescence decay rate.

For type 2 blinking, the intensity fluctuates between the dimer level (D), the intermediate level (IM) and the background level (B), Fig. 6b. Here, we have identified two possible mechanisms: First, the energy transfer rate between the neutral PBI unit and the reversibly formed radical anion varies in time. Then, a large energy transfer rate (with respect to the fluorescence decay rate) leads to complete quenching of the emission to the B level (analogous to type 1 blinking), whereas a small energy transfer rate allows to observe the IM level, i.e. the emission from the remaining neutral PBI unit. Second, type 2 blinking can be interpreted by assuming a non-fluctuating and small energy transfer rate between the neutral PBI and the radical anion. In this situation, blinking to the background level can only be observed if both monomer units are photoreduced at the same time. 

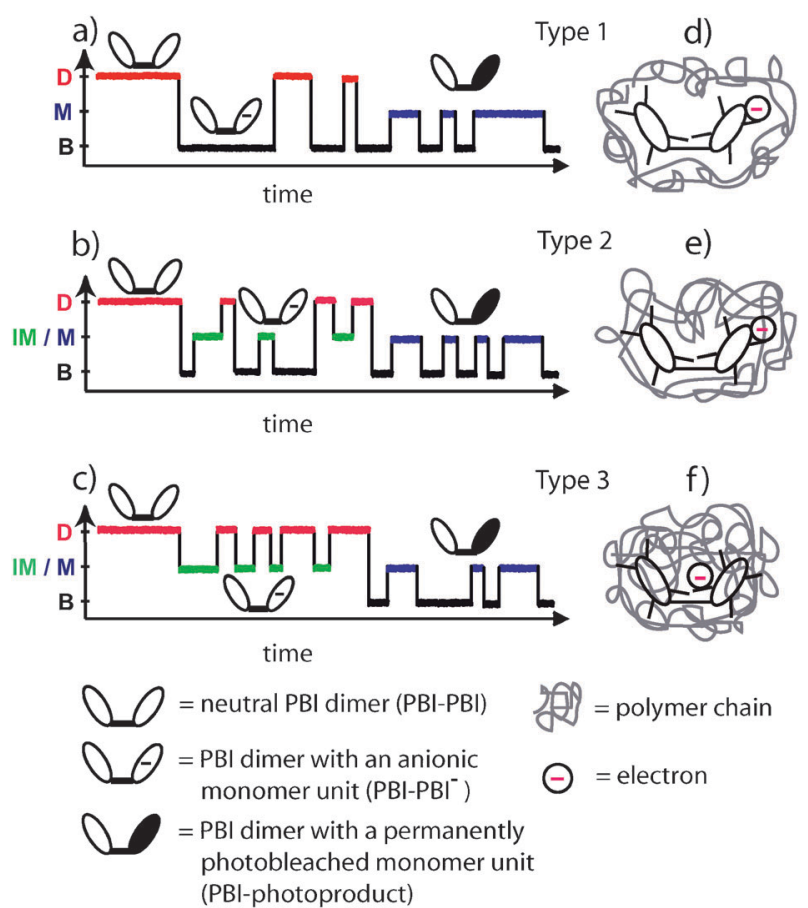

Fig. 6 Schematic representation of three different types of blinking for (a) type 1, (b) type 2 and (c) type 3. The colours encode the dimer level D (red), the intermediate level IM (green), the monomer level M (blue), and the background level B (black). The schematic representation of the species assigned to different signal levels are explained in the legends. (d)-(f) Illustrations of the local environments of the dimers causing three different types of blinking.

For type 3 blinking one of the monomer units always resides in the 'on' state, hence the signal fluctuates only between the dimer (D) and the intermediate level (IM), Fig. 6c. Here the conjecture is that the $\mathrm{PBI} \rightarrow \mathrm{PBI}^{-}$energy transfer rate is small, thus allowing for the detection of the IM level. Blinking to the background level is suppressed because reduction of the remaining PBI unit to its radical anion is prevented by a high electrostatic energy barrier that cannot be overcome by a second charge (in contrast to the second case for type 2). This mechanism is reminiscent of a Coulomb blockade observed in semiconductor quantum dots. ${ }^{47}$

For all three types of blinking behaviours the monomer level (M) is observed after irreversible photobleaching of one of the PBI units. Blinking to the background level (B) is caused by reversible radicalisation of the second $\mathrm{PBI}$.

In the following sections, we will substantiate the abovementioned interpretations based on the additional information from the fluorescence lifetimes and the emission spectra, and we will provide a microscopic picture of the local environment for three different types of blinking.

\subsection{Variations in the $\mathrm{PBI} \rightarrow \mathrm{PBI}^{-}$energy transfer rates}

The most striking feature in our data are the different regimes of energy transfer rates from the neutral PBI to the reversibly formed PBI-radical anion, which give rise to the various blinking behaviours. While type 1 dimers feature high transfer rates, type 2 dimers exhibit fluctuations between large and small transfer rates or alternatively small transfer rates without Coulomb blockade, and finally type 3 molecules show small transfer rates with Coulomb blockade. Generally, this transfer rate $k_{\mathrm{ET}}$ is determined by both the electronic coupling and the energy mismatch between the subunits of the dimer. In order to distinguish these quantities for the $\mathrm{PBI}-\mathrm{PBI}^{-}$system from those for the neutral PBI-PBI dimer as discussed above the former will be referred to as $V^{\prime}$ for the coupling and $\Delta^{\prime}$ for the energy mismatch. $V^{\prime}$ depends on the mutual orientation and the distance between $\mathrm{PBI}$ and $\mathrm{PBI}^{-}$, as well as on the dielectric screening due to the surrounding medium. As the dimer molecules are immobilised in a polymer matrix, fluctuations of the orientation and the distance can be neglected in good approximation. A heterogeneous dielectric nano-environment as provided by the disordered polymer film, however, can profoundly influence the energy transfer rate between $\mathrm{PBI}$ and $\mathrm{PBI}^{-}$. Dimers located at different positions in the sample, each within a particular arrangement of the surrounding polymer chains, will experience distinct dielectric screenings of $V^{\prime}$, and consequently substantially varying transfer rates $^{48}$ as observed in our experiments. At the same time, these different realisations of the local environments give rise to modifications of the electrostatic interactions of both PBI and $\mathrm{PBI}^{-}$with the matrix, which changes the corresponding transition energies. The resulting fluctuations of the site energy mismatch $\Delta^{\prime}$ will also contribute to the observed differences in the transfer rates. Therefore, a subtle interplay between dielectric screening of the Coulomb coupling and the interactions with the local matrix determines the actual $\mathrm{PBI} \rightarrow \mathrm{PBI}^{-}$energy transfer rate and thus the blinking behaviour for each individual dimer molecule.

The decreasing transfer rates from type 1 to type 3 suggest that the dielectric screening of $V^{\prime}$ increases. We propose therefore that the polystyrene free volume around the dimers decreases from type 1 to type 3 blinking, Fig. $6 \mathrm{~d}-\mathrm{f}$. Accordingly, the electrostatic interactions between the matrix and the PBI-monomers become stronger, which may additionally enhance the energetic mismatch such that energy transfer is further slowed down. These modulations of the transition energies occur probably mainly at the anionic site, because the electrostatic interactions between a PBI-radical and polystyrene are stronger than those between a neutral monomer and the matrix.

The possibly time-dependent transfer rates observed for type 2 blinking may be caused by thermally activated motions or rearrangements of segments of the surrounding polystyrene chains, which lead to temporal variations of the dielectric screening. These fluctuations in the local matrix can then induce conformational dynamics of the phenoxy-groups at the bay positions as well as of the flexible butyl-chains at the imidegroups of PBI, which are known to couple particularly strongly to polymer matrices. ${ }^{49-51}$ This will give rise to (temporal) changes of local interactions and thereby modify the transition energies, i.e. the energy mismatch. As a consequence, the transfer rates become time dependent, or in other words, we observe dynamic quenching of the IM level for type 2 by fast energy transfer to $\mathrm{PBI}^{-}$. This is corroborated by our timeresolved measurements, because the lifetime distribution of the IM level for type 2 is very broad (Fig. 5b, green open bars) and features clearly more entries at shorter lifetimes with respect to the lifetime distribution found for the IM level of type 3 (Fig. 5c, green open bars). An order of magnitude estimate 
demonstrates that substantial (time dependent) variations of the transfer rates are possible. If we assume that the longest observed lifetime of $8.3 \mathrm{~ns}$ represents the radiative lifetime $\tau_{\mathrm{r}}$, we can estimate the limiting values for the energy transfer rate from $k_{\mathrm{ET}}^{\text {fast, slow }}=\frac{1}{\tau_{\mathrm{ET}}^{\text {short long }}}-\frac{1}{\tau_{\mathrm{r}}}$. Here $\tau_{\mathrm{ET}}^{\text {short, long }}$ refers to the shortest (1.95 ns) and the longest ( $8 \mathrm{~ns})$ lifetime observed for

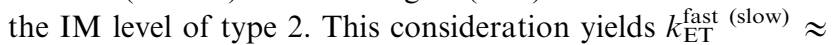
$3.9 \times 10^{8} \mathrm{~s}^{-1}\left(0.045 \times 10^{8} \mathrm{~s}^{-1}\right)$, which differ by two orders of magnitude and thus support the dynamic quenching model for type 2 blinking. Further evidence for this model for type 2 dimers in the IM level comes from the observation of continuous variations of intensity and lifetimes, which are also correlated (Fig. 4c and d green and Fig. S2, ESI $\dagger$ ): quenching of the excited state of the neutral PBI due to energy transfer to $\mathrm{PBI}^{-}$leads to a shorter lifetime in combination with a smaller fluorescence intensity and vice versa for the non-quenched situation.

\subsection{Coulomb blockade}

As an alternative explanation for type 2 blinking we proposed a small transfer rate without Coulomb blockade, which allows both monomers of the dimer to be photoreduced at the same time and gives rise to blinking to the B level. In our spectrally resolved experiments we observed for the D and IM levels of type 2 dimers nearly identical distributions of the $(0-0)$ emission bandwidths (Fig. 5f) as well as similar $\delta E$-values (Table 1). This suggests that for this type of blinking behaviour the presence of a charge on one of the monomers does not significantly influence the spectral characteristics of the remaining emitting PBI-unit. In particular, the spectral diffusion processes (on all time scales) are not altered. In other words, at the IM level of type 2 the local electric field produced by reduction of one of the PBIs is not sufficient to induce spectral fluctuations of the neutral PBI emission by an internal Stark-effect or by charge-induced changes in the local interactions with the polystyrene matrix by e.g. repulsion and/or attraction of polymer chain segments. Therefore, it is reasonable to assume that a charge on one of the monomer units of the dimer will not prevent photoreduction of the remaining monomer, and to conclude that Coulomb blockade is absent for type 2 dimers. However, given the broad lifetime distribution together with the correlated fluctuations of fluorescence intensity and lifetime in the IM level of type 2 we favour the dynamic quenching model for this type of blinking.

The Coulomb blockade model proposed for type 3 blinking is based on the observation that radicalization of the second neutral PBI unit at the IM level does not occur. This was substantiated by an estimate of the involved energy scales in our previous work. ${ }^{41}$ On the one hand, for a PBI-PBI ${ }^{-}$system a second electron has to overcome an electrostatic energy barrier of about $5300 \mathrm{~cm}^{-1}$ to radicalize the neutral PBI unit; on the other hand, due to our excitation conditions an excess energy of about $6160 \mathrm{~cm}^{-1}$ is available. Given the same order of magnitude of these figures Coulomb blockade cannot be excluded. Further evidence for the presence of the Coulomb blockade is provided by the spectrally resolved data. The peak of the $(0-0)$ emission bandwidth distribution at the IM level of type 3 is shifted by about $40 \mathrm{~cm}^{-1}$ to larger values with respect to that of the IM level of type 2 and the D levels of all types, see Fig. $5 \mathrm{~g}$.
Moreover, for type 3 we found $\delta E^{(\mathrm{IM})} \approx 81 \mathrm{~cm}^{-1}$ which is nearly twice as much as $\delta E^{(\mathrm{IM})}$ for type 2 blinking, and $\delta E^{(\mathrm{D})}$ for all types of blinking, see Table 1. Hence, for type 3 blinking the remaining neutral PBI unit features strong spectral diffusion on all time scales, which we attribute to an internal Stark-shift induced by the presence of a charge on the adjacent PBI unit. If we assume a distance of $2.2 \mathrm{~nm}$ between the centres of the chromophores and a homogeneous surrounding medium with a dielectric constant of $\varepsilon_{\mathrm{PS}}=2.5,{ }^{26}$ an elementary charge located at the centre of the radical anionic unit produces a local field of about $10^{8} \mathrm{~V} \mathrm{~m}^{-1}$ at the centre of the neutral PBI. Although the PBI molecules studied here are symmetrically substituted and, in the ideal case, do therefore not possess permanent dipole moments, the highly heterogeneous PS matrix reduces the molecular symmetry and hence induces a permanent dipole moment ${ }^{52}$ particularly for type 3 dimers with the smallest free volume. We find an upper limit of 3 Debye for the difference of the permanent dipole moment upon electronic transition from the ground state to the excited state ${ }^{53}$ which gives rise to a Stark-shift of about $70 \mathrm{~cm}^{-1}$. This value is in qualitative agreement with the observed shifts of the mean of the $(0-0)$ emission bandwidth distributions as well as with the differences in the $\delta E$-values. In summary, type 3 blinking can be explained consistently with a small energy transfer rate and simultaneous Coulomb blockade.

\subsection{Position of the charge and nature of the photoproduct}

The differences in the spectral diffusion characteristics in the IM levels of type 2 and type 3 dimers allow us to infer the position of the charge on the reversibly formed photoproducts. As discussed in Section 4.2, for type 2 the reversible photoreduction of one of the PBIs does not significantly influence the emission spectra of the neutral PBI, whereas for type 3 dimers the spectral diffusion is substantially enhanced in the IM level by the presence of a charge (manifested by a larger linewidth and a larger value for $\left.\delta E^{(\mathrm{IM})}\right)$. This suggests that even though the photoreduced species formed in the IM levels of types 2 and 3 are identical (a radical anion) the interactions that influence the emission properties of the remaining neutral monomer unit are altered (see Section 4.2). This might reflect that during type 2 and type 3 blinking the photoreduction takes place at different positions of the PBI molecule. For the PBI molecule it has been shown by experiments and theoretical studies that an additional electron leads to an increase of the electron density on the carbonyl oxygens of the imide group. ${ }^{9,18}$ Hence, the large (small) spectral shift in the IM level for type 3 (type 2) suggests that the additional charge resides on the carbonyl oxygen that is closest to (farthest from) the neutral PBI unit, see Fig. 6e and f. We note that we cannot completely rule out the possibility of interchromophoric distance variations between the monomers ${ }^{43}$ (scissoring) which contribute to spectral diffusion processes. However, as the dimers are immobilised in a rather rigid polymer matrix we consider this to be only a minor contribution to transition energy fluctuations.

Another intriguing question to be addressed concerns the nature of the photoproduct upon irreversible bleaching (Fig. 6a-c). As this is a dark species, it cannot be observed directly. Yet, for type 3 dimers we identified the photoproduct 
from our spectrally resolved measurements. In the IM level these dimers feature the strongest spectral diffusion caused by the presence of a charge on one of the monomers (see Section 4.2). Since for type $3 \delta E^{(\mathrm{IM})} \approx \delta E^{(\mathrm{M})}$ it is reasonable to assume that this photoproduct is as well a charged species. This is supported by the difference of about $35 \mathrm{~cm}^{-1}$ between the means of the $(0-0)$ emission bandwidths of the $M$ levels for type 2 and type 3 (see Table 1 and Fig. 5h), which agrees reasonably well with the Starkshift estimated above. This tends us to conclude that the charged photoproduct is in close vicinity of the remaining monomer. Finally, since the $\delta E^{(\mathrm{M})}$ values, which reflect the slow spectral diffusion processes, are very similar for all types of blinking we speculate that the photoproduct is always a charged species.

\subsection{Origin of lifetime variations}

Local fluctuations of the polymer chain density not only enhance the spectral diffusion but also alter the intrinsic lifetime of the PBI emitter. Type 3 blinking is attributed to small $\mathrm{PBI} \rightarrow \mathrm{PBI}^{-}$energy transfer rates together with Coulomb blockade, or in other words, the absence of dynamic quenching in the IM level, as discussed for type 2 above. However, we observe a rather broad lifetime distribution in the IM level of type 3 with respect to that of the D level (Fig. 5c). This indicates that other mechanisms influence the lifetime of the neutral PBI unit. For the molecule studied here it is known that neither of the perylene fragments exhibits a flat $\pi$-electron system but rather a twisted conformation with a torsional angle of $29.4^{\circ}$ between the naphthalene half units. ${ }^{43}$ As the excited state lifetime of such twisted molecules is very susceptible to small changes in the local environment, ${ }^{28,54}$ the broad lifetime distribution in the IM level of type 3 can be partly attributed to (thermally activated) rearrangements of the polymer chain around the dimer. Moreover, these segmental motions may also enforce changes in the twist angle and the orientation of the phenoxy substituents with respect to the PBI scaffold, ${ }^{20}$ thereby modifying the lifetime. In particular, the entries at longer lifetimes for the IM distribution with respect to the D distribution (Fig. 5c) indicate an enhancement of the twist angle of the remaining PBI, resulting in an increased excited state lifetime. ${ }^{54}$ This picture is consistent with the interpretation that the polystyrene free volume around type 3 dimers is the smallest and thus has a large impact on the excited state lifetime. Finally, for such molecules a further contribution to the broad lifetime distribution may come from changes in the free volume caused by electrostatic attraction and/or repulsion of the polymer segments towards or away from the radical anion. This alters the local field strength and thus modifies the spontaneous emission rate. ${ }^{26,29,55}$

The lifetime distributions of the $\mathrm{M}$ levels for type 1 and type 3 are shifted towards shorter lifetimes with respect to the corresponding distributions of the D levels, Fig. 5d. This is consistent with the conjecture made earlier ${ }^{41}$ that the emitter with the longer fluorescence lifetime bleaches first. Apparently this is not the case for type 2 , which remains unclear by now.

\section{Summary}

In the following, we summarise the main results of the paper and provide a microscopic picture for the local environments of the PBI dimers that lead to the different types of blinking.

\section{Type 1}

The main feature of type 1 blinking is the complete quenching of the IM level due to the reversible reduction of one of the PBI units to the radical anion followed by an efficient $\mathrm{PBI} \rightarrow \mathrm{PBI}^{-}$ energy transfer process. Therefore, the detectable signal levels in type 1 intensity trajectories are restricted to D and $\mathrm{M}$. This implies that during type 1 blinking, repeated charging and neutralization of one of the PBI units hardly influence the energy transfer rate. Since this rate can be altered by interactions with the local polymer matrix (see the discussion in 4.1) and since these interactions appear to be weak, we assign these dimers to those residing in a large polymer cavity, Fig. 6d.

\section{Type 2}

During type 2 blinking the signal fluctuates between the D, IM and $\mathrm{M}$ levels. The lifetime data at the IM level provide evidence that a dynamic quenching process due to a timedependent $\mathrm{PBI} \rightarrow \mathrm{PBI}^{-}$energy transfer rate is effective. The dynamic quenching process is associated mainly with the segmental dynamics of the surrounding PS chains, which suggests that dimers showing type 2 blinking are located in a smaller polymer cavity than the type 1 dimers. This is schematically shown in Fig. 6e. The spectral diffusion in the IM level is basically identical to the behaviour in the D level, and thus Coulomb blockade is not relevant here. This indicates that a charge on one of the monomers does not significantly influence the remaining neutral PBI unit and therefore we propose that the charge resides on the most distant carbonyl oxygen with respect to the neutral PBI.

\section{Type 3}

The most prominent signature of type 3 blinking is that the signal fluctuates only between the D and IM levels. This indicates that the PBI $\rightarrow \mathrm{PBI}^{-}$energy transfer is slow with respect to the fluorescence rate. This is ascribed to a large dielectric screening of the Coulomb interaction due to a very small free volume around the dimers (see Fig. 6f). The enhanced spectral diffusion in the IM level provides evidence for a strong local electrostatic field acting on the neutral PBI unit. This supports the Coulomb blockade model, suggesting that the charge resides on the closest carbonyl oxygen with respect to the neutral PBI unit.

\section{Acknowledgements}

We gratefully acknowledge the Elitenetzwerk Bayern (ENB), Deutsche Forschungsgemeinschaft (DFG GRK 1640) and the Bayreuther Institut für Makromolekülforschung (BIMF) for financial support.

\section{References}

1 F. C. de Schryver, T. Vosch, M. Cotlet, M. van de Auweraer, K. Müllen and J. Hofkens, Acc. Chem. Res., 2005, 38, 514-522.

2 H. Langhals, Helv. Chim. Acta, 2005, 88, 1309-1343.

3 F. Würthner, T. E. Kaiser and C. R. Saha-Möller, Angew. Chem., Int. Ed., 2011, 50, 3376-3410.

4 A. Sautter, B. K. Kaletas, D. G. Schmid, R. Dobrawa, M. Zimine, G. Jung, I. H. M. van Stokkum, L. de Cola, R. M. Williams and F. Würthner, J. Am. Chem. Soc., 2005, 127, 6719-6729. 
5 X. Zhan, A. Facchetti, S. Barlow, T. J. Marks, M. A. Ratner, M. R. Wasielewski and S. R. Marder, Adv. Mater., 2011, 23, 268-284.

6 J. Pan, W. Zhu, S. Li, W. Zeng, Y. Cao and H. Tian, Polymer, 2005, 46, 7658-7669.

7 R. Métivier, F. Kulzer, T. Weil, K. Müllen and T. Basché, J. Am. Chem. Soc., 2004, 126, 14364-14365.

8 E. Lang, A. Sorokin, M. Drechsler, Y. V. Malyukin and J. Köhler, Nano Lett., 2005, 5, 2635-2640.

9 A. Pron, R. R. Reghu, R. Rybakiewicz, H. Cybulski, D. Djurado, J. V. Grazulevicius, M. Zagorska, I. Kulszewicz-Bajer and J.-M. Verilhac, J. Phys. Chem. C, 2011, 115, 15008-15017.

10 D. M. Eisele, J. Knoester, S. Kirstein, J. P. Rabe and D. A. Vanden Bout, Nat. Nanotechnol., 2009, 4, 658-663.

11 F. Würthner, Chem. Commun., 2004, 1564-1579.

12 E. Lang, R. Hildner, H. Engelke, P. Osswald, F. Würthner and J. Köhler, ChemPhysChem, 2007, 8, 1487-1496.

13 C. C. Hofmann, S. M. Lindner, M. Ruppert, A. Hirsch, S. A. Haque, M. Thelakkat and J. Köhler, J. Phys. Chem. B, 2010, 114, 9148-9156.

14 J. Hernando, E. M. H. P. van Dijk, J. P. Hoogenboom, J.-J. Garcia-Lopez, D. N. Reinhoudt, M. Crego-Calama, M. F. Garcia-Parajo and N. F. van Hulst, Phys. Rev. Lett., 2006, 97, 216403

15 M. Lippitz, C. G. Hübner, T. Christ, H. Eichner, P. Bordat, A. Herrmann, K. Müllen and T. Basché, Phys. Rev. Lett., 2004, 92, 1030011-1030014.

16 H. Yoo, J. Yang, A. Yousef, M. R. Wasielewski and D. Kim, J. Am. Chem. Soc., 2010, 132, 3939-3944.

17 E. Lang, F. Würthner and J. Köhler, ChemPhysChem, 2005, 6, 935-941.

18 S. K. Lee, Y. Zu, A. Herrmann, Y. Geerts, K. Müllen and A. J. Bard, J. Am. Chem. Soc., 1999, 121, 3513-3520.

19 F. Würthner, Pure Appl. Chem., 2006, 78, 2341-2349.

20 E. Fron, G. Schweitzer, P. Osswald, F. Würthner, P. Marsal, D. Beljonne, K. Müllen, F. C. de Schryver and M. van der Auweraer, Photochem. Photobiol. Sci., 2008, 7, 1509-1521.

21 P. Tinnefeld, J. Hofkens, D.-P. Herten, S. Masuo, T. Vosch, M. Cotlet, S. Habuchi, K. Müllen, F. C. de Schryver and M. Sauer, ChemPhysChem, 2004, 5, 1786-1790.

22 F. Schindler, J. Jacob, A. C. Grimsdale, U. Scherf, K. Müllen, J. M. Lupton and J. Feldmann, Angew. Chem., Int. Ed., 2005, 44, $1520-1525$.

23 R. Hildner, U. Lemmer, U. Scherf, M. van Heel and J. Köhler, Adv. Mater., 2007, 19, 1978-1982.

24 R. Métivier, F. Nolde, K. Müllen and T. Basché, Phys. Rev. Lett., 2007, 98, 047802 .

25 J. Hernando, J. P. Hoogenboom, E. M. H. P. van Dijk, J. J. Garcia-Lopez, M. Crego-Calama, D. N. Reinhoudt, N. F. van Hulst and M. F. Garcia-Parajo, Phys. Rev. Lett., 2004, 93, 236404

26 R. A. L. Vallée, N. Tomczak, L. Kuipers, G. J. Vancso and N. F. van Hulst, Phys. Rev. Lett., 2003, 91, 038301.

27 H. Yoo, H. W. Bahng, M. R. Wasielewski and D. Kim, Phys. Chem. Chem. Phys., 2012, 14, 2001-2007.

28 R. A. L. Vallée, M. Cotlet, M. van der Auwearer, J. Hofkens, K. Müllen and F. C. de Schryver, J. Am. Chem. Soc., 2004, 126 , 2296-2297.

29 R. A. Vallée, M. Baruah, J. Hofkens, F. C. de Schryver, N. Boens and M. van der Auweraer, J. Chem. Phys., 2007, 126, 184902.
30 M. Nirmal, B. O. Dabbousi, M. G. Bawendi, J. J. Macklin, J. K. Trautman, T. D. Harris and L. E. Brus, Nature, 1996, 383, 802-804.

31 R. Verberk, A. M. van Oijen and M. Orrit, Phys. Rev. B, 2002, 66, 233202.

32 A. Issac, C. von Borczyskowski and F. Cichos, Phys. Rev. B, 2005, 71, 161302.

33 F. Cichos, C. von Borczyskowski and M. Orrit, Curr. Opin. Colloid Interf. Sci., 2007, 12, 272-284.

34 J. P. Hoogenboom, E. van Dijk, J. Hernando, N. F. van Hulst and M. F. Garcia-Parajo, Phys. Rev. Lett., 2005, 95, 097401.

35 M. Haase, C. G. Hübner, E. Reuther, A. Herrmann, K. Müllen and T. Basché, J. Phys. Chem. B, 2004, 108, 10445-10450.

36 H. Lin, S. R. Tabaei, D. Thomsson, O. Mirzov, P.-O. Larsson and I. G. Scheblykin, J. Am. Chem. Soc., 2008, 130, 7042-7051.

37 S. Unterkofler, T. Pflock, J. Southall, R. J. Cogdell and J. Köhler, ChemPhysChem, 2011, 12, 711-716.

38 E. K. L. Yeow, S. M. Melnikov, T. D. M. Bell, F. C. De Schryver and J. Hofkens, J. Phys. Chem. A, 2006, 110, 1726-1734.

39 J. Tang and R. A. Marcus, J. Chem. Phys., 2005, 123, 054704.

40 J. N. Clifford, T. D. M. Bell, P. Tinnefeld, M. Heilemann, S. M. Melnikov, J. Hotta, M. Sliwa, P. Dedecker, M. Sauer, J. Hofkens and E. K. L. Yeow, J. Phys. Chem. B, 2007, 111, 6987-6991.

41 D. Ernst, R. Hildner, C. Hippius, F. Würthner and J. Köhler, Chem. Phys. Lett., 2009, 482, 93-98.

42 C. Hippius, I. H. M. van Stokkum, E. Zangrando, R. M. Williams and F. Würthner, J. Phys. Chem. C, 2007, 111, 13988-13996.

43 C. Hippius, I. H. M. van Stokkum, E. Zangrando, R. M. Williams, M. Wykes, D. Beljonne and F. Würthner, J. Phys. Chem. C, 2008, 112, 14626-14638.

44 E. Fron, T. D. M. Bell, A. van Vooren, G. Schweitzer, J. Cornil, D. Beljonne, P. Toele, J. Jacob, K. Müllen, J. Hofkens, M. van der Auweraer and F. C. de Schryver, J. Am. Chem. Soc., 2007, 129, 610-619.

45 T. Pullerits, O. Mirzov and I. G. Scheblykin, J. Phys. Chem. B, 2005, 109, 19099-19107.

46 C. Hofmann, T. J. Aartsma, H. Michel and J. Köhler, Proc. Natl. Acad. Sci. U. S. A., 2003, 100, 15534-15538.

47 A. T. Tilke, F. C. Simmel, R. H. Blick, H. Lorenz and J. P. Kotthaus, Prog. Quantum Electron., 2001, 25, 97-138.

48 C. Curutchet, J. Kongsted, A. Muňoz-Losa, H. Hossein-Nejad, G. D. Scholes and B. Mennucci, J. Am. Chem. Soc., 2011, 133, 3078-3084.

49 S. Krause, P. F. Aramendia, D. Täuber and C. von Borczyskowski, Phys. Chem. Chem. Phys., 2011, 13, 1754-1761.

50 M. Cotlet, S. Masuo, G. Luo, J. Hofkens, M. van der Auweraer, J. Verhoeven, K. Müllen, X. S. Xie and F. de Schryver, Proc. Natl. Acad. Sci. U. S. A., 2004, 101, 14343-14348.

51 T. D. M. Bell, A. Stefan, S. Masuo, T. Vosch, M. Lor, M. Cotlet, J. Hofkens, S. Bernhardt, K. Müllen, M. van der Auweraer, J. W. Verhoeven and F. C. de Schryver, ChemPhysChem, 2005, 6, 942-948.

52 U. Bogner, P. Schätz, R. Seel and M. Maier, Chem. Phys. Lett., 1983, 102, 267-271.

53 A. S. Lukas, Y. Zhao, S. E. Miller and M. R. Wasielewski, J. Phys. Chem. B, 2002, 106, 1299-1306.

54 J. Hofkens, T. Vosch, M. Maus, F. Köhn, M. Cotlet, T. Weil, A. Herrmann, K. Müllen and F. C. de Schryver, Chem. Phys. Lett., 2001, 333, 255-263.

55 D. Wöll, E. Braeken, A. Deres, F. C. de Schryver, H. Uji-i and J. Hofkens, Chem. Soc. Rev., 2009, 38, 313-328. 